\title{
Profit sharing in unique Nash equilibrium: characterization in the two-agent case
}

\author{
by Justin LEROUX
}

\section{Cahier de recherche $n^{\circ}$ IEA-06-11}

October 2006

ISSN : 0825-8643

Copyright (C) 2006 HEC Montréal.

Tous droits réservés pour tous pays. Toute traduction ou toute reproduction sous quelque forme que ce soit est interdite. Les textes publiés dans la série des Cahiers de recherche HEC n'engagent que la responsabilité de leurs auteurs.

La publication de ce Cahier de recherche a été rendue possible grâce à des subventions d'aide à la publication et à la diffusion de la recherche provenant des fonds de l'École des HEC.

Direction de la recherche, HEC Montréal, 3000, chemin de la Côte-Sainte-Catherine, Montréal (Québec) Canada H3T 2 A7. 


\title{
Profit sharing in unique Nash equilibrium: characterization in the two-agent case
}

\author{
Justin Leroux*
}

\author{
Institut d'Économie Appliquée \\ HEC Montréal \\ 3000, chemin de la côte Sainte-Catherine \\ Montréal, QC H3T 2A7 \\ CANADA \\ e-mail: justin.leroux@hec.ca \\ Phone: (+1) 514-340-6864 \\ Fax: (+1) 514-340-6469
}

*I thank Hervé Moulin for a stimulating conversation as well as an anonymous referee for sharp comments leading to an improved version of this work. 


\begin{abstract}
Two agents jointly operate a decreasing marginal returns technology to produce a private good. We characterize the class of output-sharing rules for which the labor-supply game has a unique Nash equilibrium. It consists of two families: rules of the serial type which protect a small user from the negative externality imposed by a large user, and rules of the reverse serial type, where one agent effectively employs the other agent's labor. Exactly two rules satisfy symmetry; a result in sharp contrast with Moulin and Shenker's (Econometrica, 1992) characterization of their serial mechanism as the unique cost-sharing rule satisfying the same incentives property. We also show that the familiar stand alone test characterizes the class of fixed-path methods (Friedman, Economic Theory, 2002) under our incentives criterion.
\end{abstract}

Keywords: Joint production, serial rule, decreasing serial rule, strategyproofness. JEL classification numbers: C72, D23, D62. 


\section{Introduction}

When several producers jointly operate a production process, total output (or profits) must be shared as a function of their individual contributions (see Israelsen, 1980; Sen, 1966; Weitzman, 1974). This question applies whether the production structure is one of common access to the production function (as in the so-called "commons problem") or one where property rights to the technology are clearly defined. An extreme example of the latter is that of a monopolist hiring workers.

The production possibilities are common knowledge and exhibit decreasing marginal returns, but the individual leisure-consumption trade-offs are private information. We are concerned with sharing rules with very strong incentives properties, so as to avoid undesirable phenomena like free-riding or the familiar "tragedy of the commons". We demand that the input supply game induced by the sharing rule, in which agents decide how much input to contribute to the production process, admits a unique Nash equilibrium at all preference profiles. We refer to this incentives criterion as unique Nash incentive compatibility (UNIC). This criterion is in fact stronger than that of strategyproofness (SP), under which it is a dominant strategy for every agent to behave according to her true preference. Like SP, UNIC does not hinge on any informational assumptions and is therefore more robust than, say, Bayesian incentive compatibility. ${ }^{1}$

In the two-agent case, we characterize the class of sharing rules which are monotonic (each agent's share is increasing in her own input contribution), smooth (the sharing rule is continuously differentiable in inputs) and which satisfy UNIC (Theorem 1). This class of sharing rules is made up of two families which we call the "serial" family and the "reverse serial" family. An essential feature of rules in the serial family is that the share of a relatively small supplier of input is unaffected by changes in the supply level of a large supplier (a feature called the "serial principle" in Sprumont, 1998) while the converse is true for rules of the reverse serial type: the share of a large supplier is unaffected by changes in the input level of a small supplier.

In addition, the externality imposed by a small user on large users is typically negative under a serial rule. Conversely, a large supplier of input typically imposes positive externalities on small suppliers under a reverse serial rule. Thus, with the exception of priority rules (which belong to both families), we argue that serial rules are more adapted to the commons problem, with the negative externality reflecting congestion, while reverse serial rules correspond to a more "corporate" production structure where the owner of the facility extracts rents from the labor contribution of a worker.

In Section 5 we consider a popular axiom in the commons literature. The stand alone test (SA) captures the essence of the commons problem by demanding that no agent be made better off by the presence of others than if she were operating the technology by herself (see, e.g., Moulin and Shenker, 1992; Suh, 1997; Sprumont, 1998; Hougaard and Thorlund-Pertersen, 2000). It turns out that SA characterizes the output-sharing version of the class of fixed-path methods (FPMs) discussed in Friedman $(2002,2004)$ (see Theorem 2). These sharing rules allocate marginal quantities of input, and the corresponding amounts of output, along a prespecified path in the agents' input space. Among well-known FPMs are the Moulin and Shenker serial rule and priority rules, which follow the diagonal of the positive orthant and an axis of the agents' input space, respectively.

\footnotetext{
${ }^{1}$ We refer the reader to a companion paper (Leroux, 2006) for a discussion of strategyproofness and UNIC relative to Bayesian incentive compatibility.
} 
After relating our work to the existing literature (Section 2) we define the serial and reverse serial families of sharing rules (Section 3) and state our main characterization theorem (Theorem 1) in Section 4. In Section 5 we characterize the class of FPMs by SA and UNIC (Theorem 2).

\section{Relation to the literature}

This work contributes to the large literature on the trade-off between efficiency and incentive compatibility.

Because mechanisms in the serial and reverse serial family satisfy UNIC, they fail to be first-best efficient (see Leroux, 2004). In fact, when coupled with the requirement that agents be treated anonymously, UNIC is so restrictive as to pin down exactly two sharing rules (Corollary 1). These rules are the output-sharing versions of the Moulin and Shenker serial rule (see Moulin and Shenker, 1992) and the de Frutos decreasing serial rule (see de Frutos, 1998) originally formulated in the costsharing framework, where agents each demand a quantity of output and split the cost of meeting total demand. Our result is in direct contradiction with Theorem 4 in Moulin and Shenker (1992) which wrongly characterizes the surplus-sharing version of the serial rule as the only one satisfying UNIC and anonymity. The argument there consists in stating that the surplus-sharing framework is a mere rewriting of the cost-sharing one. In fact, as our result shows, it is not.

We believe that Moulin and Shenker's false result has had a significant impact on the direction taken by the subsequent literature on the joint production of private goods. Indeed, we count only two published papers framed in the surplus-sharing interpretation since 1992 (Maniquet and Sprumont, 1999, and Leroux, 2004) against many more formulated in the cost sharing context (see, for instance, Shenker, 1992; Sprumont, 1998; Friedman, 2002, 2004; Téjédo and Truchon, 2002; and Alcalde and Angel-Silva, 2004, in addition to the above references.). It seems authors have focused on cost-sharing problems with the implicit belief that all findings should carry over, unaltered, to the surplus-sharing framework. Therefore, we feel our exposing this discrepancy between the two models may help correct this unjustified inbalance.

We should mention that the fact that Theorem 4 of Moulin and Shenker (1992) is wrong can be inferred from a result in a yet unpublished paper by Suh (Suh, 1997). However, he fails to juxtapose his finding to the (false) result of Moulin and Shenker. Moreover, the aim of Suh (1997) is to classify the output-sharing versions of the Moulin and Shenker serial rule and the de Frutos decreasing serial rule with respect to their incentives and axiomatic properties: Suh does not attempt to characterize the general class of incentive compatible sharing rules as we do here.

Regardless of the framework adopted, mapping out the class of strategy-proof or UNIC mechanisms in economies with production of private goods remains a large open question. So far, authors have mainly approached it by pairing SP with additional axioms: e.g. symmetry (Moulin and Shenker, 1992; Suh, 1997), or individual rationality (Leroux, 2005). Our Theorem 1 is the first to pin down the full class of UNIC rules for the two-agent case. Characterizing this class in the many-agent case proves to be a much more difficult task. Nonetheless, our results provide insights regarding the kind of rules which one might expect to satisfy UNIC in that case. We refer the reader to a companion paper (Leroux, 2005) for some intuition behind the technical difficulties which arise with three or more agents.

The FPMs we characterize in Theorem 2 are the output-sharing versions of cost-sharing mech- 
anisms introduced Friedman (2002) as non-anonymous generalizations of the Moulin and Shenker serial rule retaining its strong incentives properties. We show in a companion paper (Leroux, 2005) that Theorem 2 does not extend to the many-agent case and discuss the appeal of FPMs in partnership problems. We show there that to each FPM corresponds an equivalent distribution of property rights to the production process, and vice versa.

Recent related literature on the common production of private goods considers weaker interpretations of incentive compatibility (see, e.g., Corchón and Puy, 2002; Shin and Suh, 1997). For instance, Corchón and Puy establish that any continuous sharing rule admits a Pareto-efficient allocation which can be Nash-implemented. Yet, any game implementing such an outcome must have several, non-welfare-equivalent Nash equilibria at some profiles. Here we insist on the uniqueness of the Nash equilibrium, a much more demanding requirement than the above kind of Nash-implementability.

\section{The two families}

Two agents jointly operate an increasing, strictly concave and continuously differentiable production function $F: \mathbb{R}_{+} \rightarrow \mathbb{R}_{+}$such that $F(0)=0$. When each agent $i$ supplies $x_{i} \geq 0$ units of input, the input vector $x=\left(x_{1}, x_{2}\right)$ yields $F\left(x_{1}+x_{2}\right)$ units of total output.

Agent $i$ 's utility from supplying $x_{i} \geq 0$ and receiving $y_{i} \geq 0$ units of output is $u_{i}\left(x_{i}, y_{i}\right)$; the utility function, $u_{i}$, is decreasing in $x_{i}$, increasing in $y_{i}$ and quasi-concave. We denote by $\mathcal{U}$ the class of utility functions. A preference profile (or a profile) is a pair $\left(u_{1}, u_{2}\right) \in \mathcal{U} \times \mathcal{U}$.

A sharing rule is a mapping $\xi: \mathbb{R}_{+}^{2} \rightarrow \mathbb{R}_{+}^{2}$ such that $\xi_{1}(x)+\xi_{2}(x)=F\left(x_{1}+x_{2}\right)$ for all $x$, which is smooth ( $\xi$ is continuously differentiable) and monotonic $\left(\frac{\partial \xi_{i}}{\partial x_{i}}>0\right.$ for $\left.i=1,2\right)$.

We denote by $\mathcal{B}$ the class of non-decreasing functions $b: \mathbb{R}_{+} \rightarrow \mathbb{R}_{+} \cup\{+\infty\}$ which are continuous on $\mathbb{R}_{+}$and increasing on $\{t \geq 0 \mid 0<b(t)<+\infty\}$. I.e., $b$ can only be constant on a range where it returns zero or infinity; also, its graph can have a vertical asymptote. We denote by $\mathcal{F}$ the class of mappings from $\mathbb{R}_{+}$to itself which are increasing, strictly concave and continuously differentiable.

Definition 1 A sharing rule $\xi$ is of the serial type if there exists $b \in \mathcal{B}$ and $g^{s}, h^{s} \in \mathcal{F}$ s.t.

$$
\xi(x)= \begin{cases}\left(g^{s}\left(x_{1}\right), F\left(x_{1}+x_{2}\right)-g^{s}\left(x_{1}\right)\right) & \text { if } x_{2} \geq b\left(x_{1}\right) \\ \left(F\left(x_{1}+x_{2}\right)-h^{s}\left(x_{2}\right), h^{s}\left(x_{2}\right)\right) & \text { if } x_{2} \leq b\left(x_{1}\right)\end{cases}
$$

We denote by $\mathcal{S}$ the class of such rules.

The reader will notice that when $x_{2} \geq b\left(x_{1}\right)$ agent 1 's output share is unaffected by changes in $x_{2}$ above $b\left(x_{1}\right)$; a symmetric statement holds if $x_{2} \leq b\left(x_{1}\right)$. Rules of the serial type protect low-level users of the facility from the negative externalities imposed by high-level users above a certain level.

It is easily checked that $\mathcal{S}$ includes the output-sharing version of the Moulin and Shenker serial rule (the serial rule, see Moulin and Shenker, 1992) with $b\left(x_{1}\right)=x_{1}, g^{s}(t)=h^{s}(t)=\frac{1}{2} F(2 t)$; and of the fixed-path methods discussed in Friedman $(2002,2004)$ : with $g^{s}\left(x_{1}\right)=\int_{0}^{x_{1}} F^{\prime}(t+b(t)) d t$ and

$$
h^{s}\left(x_{2}\right)= \begin{cases}F\left(x_{2}\right) & \text { if } x_{2} \leq b(0) \\ F(b(0))+\int_{b(0)}^{x_{2}} F^{\prime}\left(b^{-1}(t)+t\right) d t & \text { otherwise }\end{cases}
$$


Definition $2 A$ sharing rule $\xi$ is of the reverse serial type if there exists $b \in \mathcal{B}$ and $g^{r}, h^{r} \in \mathcal{F}$ s.t.

$$
\xi(x)= \begin{cases}\left(F\left(x_{1}+x_{2}\right)-h^{r}\left(x_{2}\right), h^{r}\left(x_{2}\right)\right) & \text { if } x_{2} \geq b\left(x_{1}\right) \\ \left(g^{r}\left(x_{1}\right), F\left(x_{1}+x_{2}\right)-g^{r}\left(x_{1}\right)\right) & \text { if } x_{2} \leq b\left(x_{1}\right)\end{cases}
$$

We denote by $\mathcal{R}$ the class of such mechanisms.

Here, however, agent 2's output level is unaffected by changes in $x_{1}$ below $b^{-1}\left(x_{2}\right)$ if $x_{2} \geq b\left(x_{1}\right){ }^{2}$ If $x_{2} \geq b\left(x_{1}\right)$, a mechanism of the reverse serial type provides a compensation schedule, $h^{r}$, for the high-level supplier of input (agent 2, "the worker") whose labor benefits the low-level supplier (agent 1, "the employer").

Clearly, the decreasing serial rule (as in de Frutos [6]) belongs to $\mathcal{R}: b\left(x_{1}\right)=x_{1}, g^{r}(t)=h^{r}(t)=$ $\frac{1}{2} F(2 t)$.

The intersection of $\mathcal{R}$ and $\mathcal{S}$ is nonempty. It is worth noting that the priority rules giving full access to one agent belong to both families: for instance, both $\left[b \equiv 0 ; g^{s}\left(x_{1}\right)=F\left(x_{1}\right)\right]$ and $[b \equiv+\infty$; $\left.g^{r}\left(x_{1}\right)=F\left(x_{1}\right)\right]$ represent the rule giving priority to agent 1 . More generally we denote by $\mathcal{D}_{1}$ (resp. $\mathcal{D}_{2}$ ) the class of rules where $b \equiv 0$ (resp. $b \equiv+\infty$ ), such that agent 1 (resp. 2) is a dictator, and by $\mathcal{D} \equiv \mathcal{D}_{1} \cup \mathcal{D}_{2}$ the class of dictatorships. The reader can easily check that $\mathcal{R} \cap \mathcal{S}=\mathcal{D}$.

\section{Main result}

Our main result is a full characterization of the class of sharing rules satisfying UNIC.

Theorem 1 Let $\xi$ be a sharing rule. The following statements are equivalent:

i) $\xi \in \mathcal{S} \cup \mathcal{R}$,

ii) the supply game associated with $\xi\left(\right.$ strategy $x_{i}$, payoff $u_{i}\left(x_{i}, \xi_{i}(x)\right)$ ) admits at most one Nash equilibrium at all profiles in $\mathcal{U} \times \mathcal{U}$,

iii) (UNIC) the supply game associated with $\xi$ admits exactly one Nash equilibrium at all profiles in $\mathcal{U} \times \mathcal{U}$.

A standard result in the implementation literature (see Dasgupta et al., 1979) implies that the direct mechanism associated with a sharing rule $\xi \in \mathcal{S} \cup \mathcal{R}$ is SP. In this mechanism, each agent reports a utility function in $\mathcal{U}$ and the outcome is the unique Nash equilibrium of the supply game under the reported profile. Moreover, the unique Nash equilibrium of the supply game turns out to be strong (easily checked), therefore the associated direct mechanism is also group-strategyproof (i.e. invulnerable to coordinated manipulations).

The following corollary follows immediately upon noticing that the only symmetric rules in $\mathcal{S} \cup \mathcal{R}$ must be associated with $b \equiv I d_{\mathbb{R}_{+}}$. Exactly two such rules exist:

Corollary 1 The output-sharing versions of the Moulin and Shenker serial rule and the de Frutos' decreasing serial rule are the only two symmetric sharing rules satisfying UNIC.

It is shown in Suh (1997) that the above corollary continues to hold in the many-agent case. Suh's approach, which consists in comparing properties of the Moulin and Shenker serial rule and the de

\footnotetext{
${ }^{2}$ Note that from the definition of $\mathcal{B}, b^{-1}\left(x_{2}\right)$ exists for any positive $x_{2}$ in the range of $b$.
} 
Frutos decreasing serial rule, is quite different from ours as he does not attempt to characterize the class of incentive compatible sharing rules. While Suh's result is interesting, restricting attention to symmetric rules can be overly reductive when the production function arises from (possibly) unequal capital contributions on the agents' part (see Leroux, 2005, for a discussion of asymmetric methods and their economic interpretation in terms of property rights to the production process).

We give some intuition behind the discrepancy between the cost- and output-sharing models. Define the cost function $C \equiv F^{-1}$; clearly, $C$ and $F$ are equivalent representations of the same production possibilities. A cost-sharing rule, $\zeta$, allocates to any vector of demands $\left(y_{1}, y_{2}\right) \in \mathbb{R}_{+}^{2}$ a cost vector $\left(x_{1}, x_{2}\right) \in \mathbb{R}_{+}^{2}$ such that $x_{1}+x_{2}=C\left(y_{1}+y_{2}\right)$. The Moulin and Shenker serial rule, defined by

$$
\zeta_{i}^{M S}(y)=\frac{1}{2} C\left(2 y_{i}\right) \quad \text { and } \quad \zeta_{j}^{M S}(y)=C\left(y_{1}+y_{2}\right)-\frac{1}{2} C\left(2 y_{i}\right)
$$

if $y_{i} \leq y_{j}$, is the only cost-sharing rule whose associated demand game has a unique Nash equilibrium at all profiles (Theorem 2 in Moulin and Shenker, 1992). In particular, the de Frutos decreasing serial cost-sharing rule, defined by

$$
\zeta_{i}^{d F}(y)=\frac{1}{2} C\left(2 y_{j}\right) \quad \text { and } \quad \zeta_{j}^{d F}(y)=C\left(y_{1}+y_{2}\right)-\frac{1}{2} C\left(2 y_{j}\right)
$$

if $y_{i} \leq y_{j}$, is not well defined when $C$ is strictly convex (i.e. when $F$ is strictly concave, as in our framework) as it does not guarantee positive cost shares. This fact, in light of Corollary 1 , suggests that the difference between the cost- and output-sharing versions of the sharing problem goes beyond simple rewriting.

\section{The stand alone test}

Given that $F$ exhibits decreasing marginal returns, the context is one of negative externalities where the participation of each agent decreases the productivity of the others. In a commons problem the mechanism designer may require that the sharing rule reflects these negative externalities. We propose the following interpretation of this requirement.

The stand alone test (SA) A sharing rule, $\xi$, satisfies SA if and only if

$$
\xi_{i}(x) \leq F\left(x_{i}\right)
$$

for all $x \in \mathbb{R}_{+}^{2}$ and $i=1,2$.

SA asks that no agent benefits from the presence of the other agent. We show that it characterizes the output-sharing versions of the class of fixed-path methods (Friedman, 2002) among rules of the serial and reverse serial family. Fixed-path methods allocate marginal increments of input-and the corresponding amount of output - along a prespecified continuous increasing path in the agents' input space. With our notations, the class of fixed-path methods consists of the two priority rules as well as some non-dictatorial serial rules:

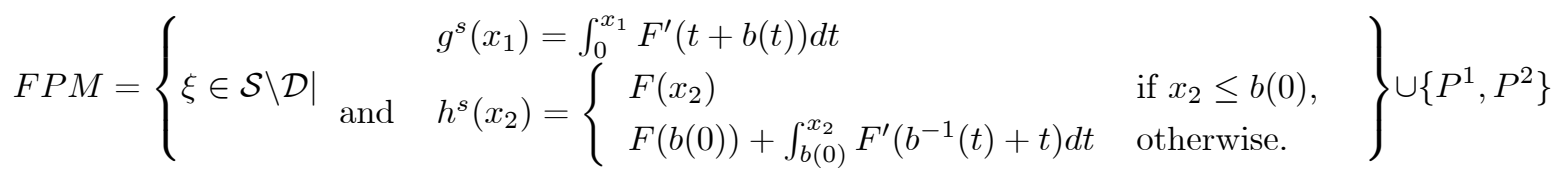


where $P_{i}^{i}(x)=F\left(x_{i}\right)$ and $P_{j}^{i}=F\left(x_{i}+x_{j}\right)-F\left(x_{i}\right) .^{3}$

Theorem 2 Let $\xi \in \mathcal{S} \cup \mathcal{R}$, the following statements are equivalent:

i) $\xi \in F P M$,

ii) $\xi$ satisfies $S A$.

Proof. Notation: Define $X_{1}=\left\{x_{1}>0 \mid 0<b\left(x_{1}\right)<+\infty\right\}$ and write $\left.X_{1}=\right] \underline{x}_{1}, \bar{x}_{1}[$. Notice that $X_{1} \neq \emptyset$ if and only if $\xi \notin \mathcal{D}$.

i) $\Longrightarrow$ ii) Suppose $\xi \in F P M$. Consider the following property:

Cross Monotonicity (CM) A sharing rule, $\xi$, satisfies CM if and only if

$$
\frac{\partial \xi_{i}}{\partial x_{j}} \leq 0
$$

on $\mathbb{R}_{+}^{2}$ for $i \neq j$.

Because CM is more demanding than SA, it suffices to show that $\xi$ satisfies CM. ${ }^{4}$ If $\xi=P^{1}$, then clearly $\frac{\partial \xi_{1}}{\partial x_{2}} \equiv 0$ and $\frac{\partial \xi_{2}}{\partial x_{1}}(x)=F^{\prime}\left(x_{1}+x_{2}\right)-F^{\prime}\left(x_{1}\right) \leq 0$ for all $x$ by the concavity of $F$. Similarly, $P^{2}$ also satisfies CM.

If $\xi \in \mathcal{S} \backslash \mathcal{D}$, then

$$
\frac{\partial \xi_{1}}{\partial x_{2}}= \begin{cases}F^{\prime}\left(x_{1}+x_{2}\right)-F^{\prime}\left(b^{-1}\left(x_{2}\right)+x_{2}\right) & \text { if } x_{2} \leq b\left(x_{1}\right) \\ 0 & \text { otherwise }\end{cases}
$$

and

$$
\frac{\partial \xi_{2}}{\partial x_{1}}= \begin{cases}0 & \text { if } x_{2} \leq b\left(x_{1}\right), \\ F^{\prime}\left(x_{1}+x_{2}\right)-F^{\prime}\left(x_{1}+b\left(x_{1}\right)\right) & \text { otherwise. }\end{cases}
$$

The concavity of $F$ ensures that these cross-derivatives are non-positive.

ii) $\Longrightarrow$ i) Suppose $\xi \in \mathcal{D}_{1}$, i.e. $\xi(x)=\left(g\left(x_{1}\right), F\left(x_{1}+x_{2}\right)-g\left(x_{1}\right)\right)$ for some $g \in \mathcal{F}$. By SA, $g\left(x_{1}\right) \leq F\left(x_{1}\right)$ and $\xi_{2}\left(x_{1}, 0\right)=F\left(x_{1}\right)-g\left(x_{1}\right) \leq F(0)=0$ for all $x_{1} \geq 0$. Hence, $g \equiv F$ and $\xi=P^{1}$. Similarly, if $\xi \in \mathcal{D}_{2}$ then $\xi=P^{2}$.

Suppose $\xi \notin \mathcal{D}$, we show that $\xi \notin \mathcal{R} \backslash \mathcal{D}$. By contradiction, suppose $\xi \in \mathcal{R} \backslash \mathcal{D}$, then for any $x_{1} \in X_{1}$,

$$
\begin{array}{lll} 
& \xi_{2}(x)=F\left(x_{1}+x_{2}\right)-g^{r}\left(x_{1}\right) \leq F\left(x_{2}\right) & \text { for any } x_{2} \leq b\left(x_{1}\right) \text { by SA, } \\
\Longrightarrow \quad g^{r}\left(x_{1}\right) \geq F\left(x_{1}+x_{2}\right)-F\left(x_{2}\right) & \text { for any } x_{2} \leq b\left(x_{1}\right), \\
\Longrightarrow \quad g^{r}\left(x_{1}\right) \geq F\left(x_{1}\right) & \text { by concavity of } F, \text { choosing } x_{2}=0, \\
\Longrightarrow \quad g^{r} \equiv F \text { on } X_{1}, & \text { by SA (for agent } 1) .
\end{array}
$$

Also, by smoothness

$$
F^{\prime}\left(x_{1}+b\left(x_{1}\right)\right)=g^{r \prime}\left(x_{1}\right)
$$

for all $x_{1} \in X_{1}$ which is incompatible with both $g^{r} \equiv F$ and the strict concavity of $F$.

\footnotetext{
${ }^{3}$ The reader can easily check that $F P M \subseteq\left\{\xi \in \mathcal{S} \backslash \mathcal{D} \mid g^{s}(0)=h^{s}(0)=0\right\} \cup\left\{P^{1}, P^{2}\right\}$. We thank an anonymous referee for pointing out that this inclusion is strict.

${ }^{4}$ Under CM, budget balance and the positivity of output shares require:

$$
F\left(x_{i}\right) \geq \xi_{i}\left(x_{i}, 0\right) \geq \xi_{i}(x) \quad \text { for all } x \text { and all } i .
$$
}


Finally, we show that if $\xi \in \mathcal{S} \backslash \mathcal{D}$, then SA implies that $\xi$ is an FPM. Notice that if $0<b(0)<+\infty$, then $\xi_{2}(0,0)=h^{s}(0)=0$ and $\xi_{1}(0, b(0))=g^{s}(0)=0$. Similarly, the result holds if $\underline{x_{1}}>0$ and if $b^{-1}(\{0\})=\{0\}$. Integrating the smoothness condition,

$$
F^{\prime}\left(x_{1}+b\left(x_{1}\right)\right)=g^{s \prime}\left(x_{1}\right)=h^{s \prime}\left(b\left(x_{1}\right)\right) \quad \text { for all } x_{1} \in X_{1},
$$

between $(0,0)$ and a point $\left(x_{1}, x_{2}\right)$ on the graph of $b$ and taking into account the fact that $g^{s}(0)=$ $h^{s}(0)=0$ yields:

$$
\begin{aligned}
g^{s}\left(x_{1}\right) & =\int_{0}^{x_{1}} F^{\prime}(t+b(t)) d t \\
\text { and } \quad h^{s}\left(x_{2}\right) & =F(b(0))+\int_{b(0)}^{x_{2}} F^{\prime}\left(b^{-1}(t)+t\right) d t \quad \text { if } x_{2} \geq b(0) .
\end{aligned}
$$

The proof is complete upon noticing that SA applied to agent 1 implies $h^{s}\left(x_{2}\right)=F\left(x_{2}\right)$ for any $x_{2} \leq b(0)$ (consider the profile $\left(0, x_{2}\right)$ ).

\section{Concluding comment}

As recalled in Section 2, most of the existing literature on cooperative production is framed in the cost-sharing context, with the general intuition that the output-sharing problem is a mere rewriting of the cost-sharing problem (see, e.g., Section 8 of Moulin and Shenker, 1992). However, our Corollary 1 contrasts markedly with this view and exposes an error in a very influential article by Moulin and Shenker which we suspect has been responsible for the direction taken by the subsequent literature on the management of joint production processes.

This discrepancy between these two formulations of the same problem is reminiscent of a somewhat different finding in Moulin and Watts (1997). They show that if given the choice between playing the average cost game or the average returns game, individuals would unambiguously choose the latter. We deem worthy of exploration whether adopting the cost- or output-sharing framework has other practical consequences.

\section{A Appendix}

\section{A.1 Proof of Theorem 1}

i) $\Longrightarrow$ iii) The proofs of the strategic properties of the Moulin and Shenker serial rule (See Moulin and Shenker, 1992, Theorem 1) and of fixed-path methods (see Friedman, 2002) in the cost-sharing context can be adapted to our setting. We nonetheless provide a proof for the sake of completeness.

We will use the following lemma extensively; its obvious proof is omitted.

Lemma 1 Suppose $f, g \in \mathcal{F}$ coincide on the interval $\left[\lambda^{-}, \lambda^{+}\right]$, and for any utility function, $u$, denote $\lambda_{1}=\max _{\lambda} u(\lambda, f(\lambda))$ and $\lambda_{2}=\max _{\lambda} u(\lambda, g(\lambda))$. The following statements are true:

- $\lambda_{1}<\lambda^{-}$iff $\lambda_{2}<\lambda^{-}$,

- $\lambda_{1}>\lambda^{+}$iff $\lambda_{2}>\lambda^{+}$,

- $\forall \lambda \in\left[\lambda^{-}, \lambda^{+}\right], \lambda_{1}=\lambda$ iff $\lambda_{2}=\lambda$. 
The existence of a Nash equilibrium of the supply game is guaranteed by the convexity of preferences and the (easily checked) fact that for any $x$, the boundary of each agent's option set is the graph of a strictly concave function: $\xi_{1}\left(\cdot, x_{2}\right)$ and $\xi_{2}\left(x_{1}, \cdot\right)$, respectively. Each agent has a unique best response to the other agent's strategy.

We show uniqueness by contradiction. Fix a preference profile, and suppose the supply game admits two distinct Nash equilibria, $x^{*}$ and $x^{* *}$, at that profile. We claim that $\left[b\left(x_{1}^{*}\right) \leq x_{2}^{*} \Longrightarrow b\left(x_{1}^{* *}\right)>x_{2}^{* *}\right]$ and $\left[b\left(x_{1}^{*}\right) \geq x_{2}^{*} \Longrightarrow b\left(x_{1}^{* *}\right)<x_{2}^{* *}\right]$. Suppose not, and suppose without loss of generality that $b\left(x_{1}^{*}\right) \leq x_{2}^{*}$ and $b\left(x_{1}^{* *}\right) \leq x_{2}^{* *}$. Then, if $\xi \in \mathcal{R}$, observe that $\xi_{2}\left(x_{1}^{*}, \cdot\right) \equiv \xi_{2}\left(x_{1}^{* *}, \cdot\right) \equiv h^{r}(\cdot)$ on the interval $\left[\max \left\{b\left(x_{1}^{*}\right), b\left(x_{1}^{* *}\right)\right\},+\infty\right.$ [ (and therefore on any closed subinterval). By Lemma $1, x_{2}^{*}=x_{2}^{* *}$. Because agent 1 has a unique best response to $x_{2}^{*}$ (and to $x_{2}^{* *}$ ) it follows that $x_{1}^{*}=x_{1}^{* *}$, contradicting the assumption that $x^{*}$ and $x^{* *}$ are distinct. If $\xi \in \mathcal{S}$, the argument is similar upon noticing that $\xi_{1}\left(\cdot, x_{2}^{*}\right) \equiv \xi_{1}\left(\cdot, x_{2}^{* *}\right) \equiv g^{s}(\cdot)$ on the interval $\left[0, \min \left\{x_{2}^{*}, x_{2}^{* *}\right\}\right]$.

It follows from the argument of the previous paragraph that the supply game induced by $\xi$ has a unique Nash equilibrium at all profiles if $\xi \in \mathcal{D}$ since, for these sharing rules, the graph of the boundary function coincides with one of the axes. Next, we only show uniqueness for the case $\xi \in \mathcal{R} \backslash \mathcal{D}$ as the argument is similar for $\xi \in \mathcal{S} \backslash \mathcal{D}$.

Let $\xi \in \mathcal{R} \backslash \mathcal{D}$ and suppose without loss that $b\left(x_{1}^{*}\right) \leq x_{2}^{*}$ and $b\left(x_{1}^{* *}\right)>x_{2}^{* *}$. We claim that $b\left(x_{1}^{* *}\right) \geq$ $x_{2}^{*}$. If not, then $x_{2}^{*}>b\left(x_{1}^{* *}\right)>x_{2}^{* *}$. Also, $\xi_{2}\left(x_{1}^{*}, \cdot\right) \equiv \xi_{2}\left(x_{1}^{* *}, \cdot\right) \equiv h^{r}(\cdot)$ on $\left[\max \left\{b\left(x_{1}^{*}\right), b\left(x_{1}^{* *}\right)\right\},+\infty[;\right.$ see Figure 1 (drawn for the case $b\left(x_{1}^{* *}\right) \geq b\left(x_{1}^{*}\right)$ ). It follows from Lemma 1 and $x_{2}^{*} \geq \max \left\{b\left(x_{1}^{*}\right), b\left(x_{1}^{* *}\right)\right\}$ that $x_{2}^{* *} \geq b\left(x_{1}^{* *}\right)$, a contradiction of our assumption. We now show that $b\left(x_{1}^{* *}\right) \leq x_{2}^{*}$ (and hence $\left.b\left(x_{1}^{* *}\right)=x_{2}^{*}\right)$. The argument is similar: suppose $x_{2}^{*}<b\left(x_{1}^{* *}\right)$, then $b\left(x_{1}^{* *}\right)>x_{2}^{*}>b\left(x_{1}^{*}\right)$. Notice that $\xi_{1}\left(\cdot, x_{2}^{*}\right) \equiv \xi_{1}\left(\cdot, x_{2}^{* *}\right) \equiv g^{r}(\cdot)$ coincide on $\left[\max \left\{b^{-1}\left(x_{2}^{*}\right), b^{-1}\left(x_{2}^{* *}\right)\right\},+\infty\left[\right.\right.$ with $b^{-1}\left(x_{2}^{* *}\right)$ to be taken to equal to $\inf \{t>0 \mid 0<b(t)<\infty\}$ if $x_{2}^{* *}=0$. Thus, Lemma 1 implies, along with the fact that $x_{1}^{* *} \geq$ $\max \left\{b^{-1}\left(x_{2}^{*}\right), b^{-1}\left(x_{2}^{* *}\right)\right\}$, the contradiction that $b\left(x_{1}^{*}\right)=b\left(x_{1}^{* *}\right) \geq x_{2}^{*}$. The same two-part argument can be invoked to prove $b\left(x_{1}^{*}\right)=x_{2}^{* *}$. We now have $b\left(x_{1}^{* *}\right)=x_{2}^{*}>b\left(x_{1}^{*}\right)=x_{2}^{* *}$. Applying Lemma 1 once again to the fact that $\xi_{1}\left(\cdot, x_{2}^{*}\right) \equiv \xi_{1}\left(\cdot, x_{2}^{* *}\right) \equiv g^{r}(\cdot)$ coincide on $\left[\max \left\{b^{-1}\left(x_{2}^{*}\right), b^{-1}\left(x_{2}^{* *}\right)\right\},+\infty[\right.$ yields $b\left(x_{1}^{*}\right)=b\left(x_{1}^{* *}\right)=x_{2}^{*}$, a contradiction.

iii) $\Longrightarrow$ ii) Obvious.

ii) $\Longrightarrow$ i) Let $\xi$ be a sharing rule for which the associated supply game has at most one Nash equilibrium at all profiles, we show $\xi \in \mathcal{S} \cup \mathcal{R}$.

Notation: We say that a $2 \times 2$ matrix, $\left[\alpha_{i j}\right]$, is acyclic if $\alpha_{12} \alpha_{21}=0$. We say that a sharing rule, $\xi$, is acyclic at a point $x \in \mathbb{R}_{+}^{2}$ if the Jacobian matrix of $\xi$ at $x,\left[\frac{\partial \xi_{i}}{\partial x_{j}}(x)\right]$, is acyclic. We define $N E=\left\{x \in \mathbb{R}_{+}^{2} \mid \frac{\partial \xi_{1}}{\partial x_{2}}(x) \neq 0\right.$ and $\left.\frac{\partial \xi_{2}}{\partial x_{1}}(x)=0\right\}, S W=\left\{x \in \mathbb{R}_{+}^{2} \mid \frac{\partial \xi_{1}}{\partial x_{2}}(x)=0\right.$ and $\left.\frac{\partial \xi_{2}}{\partial x_{1}}(x) \neq 0\right\}$ and $D=\left\{x \in \mathbb{R}_{+}^{2} \mid \frac{\partial \xi_{1}}{\partial x_{2}}(x)=\frac{\partial \xi_{2}}{\partial x_{1}}(x)=0\right\}$.

We start the proof by restating a lemma from the proof of Theorem 2 in Moulin and Shenker (1992), which still holds in our setting. It is related to the finding that strategy-proof mechanisms must be acyclic at differentiable points (see Satterthwaite and Sonnenschein, 1981).

Lemma 2 (Lemma 5 in [12]) If the supply game associated with $\xi$ has at most one Nash equilibrium for all profiles in $\mathcal{U} \times \mathcal{U}$, then $\xi$ is acyclic at all $x \in \mathbb{R}_{+}^{2}$.

It is clear from acyclicity that $N E, S W$ and $D$ form a partition of $\mathbb{R}_{+}^{2}$ and from smoothness that $N E$ and $S W$ are open whereas $D$ is closed. 


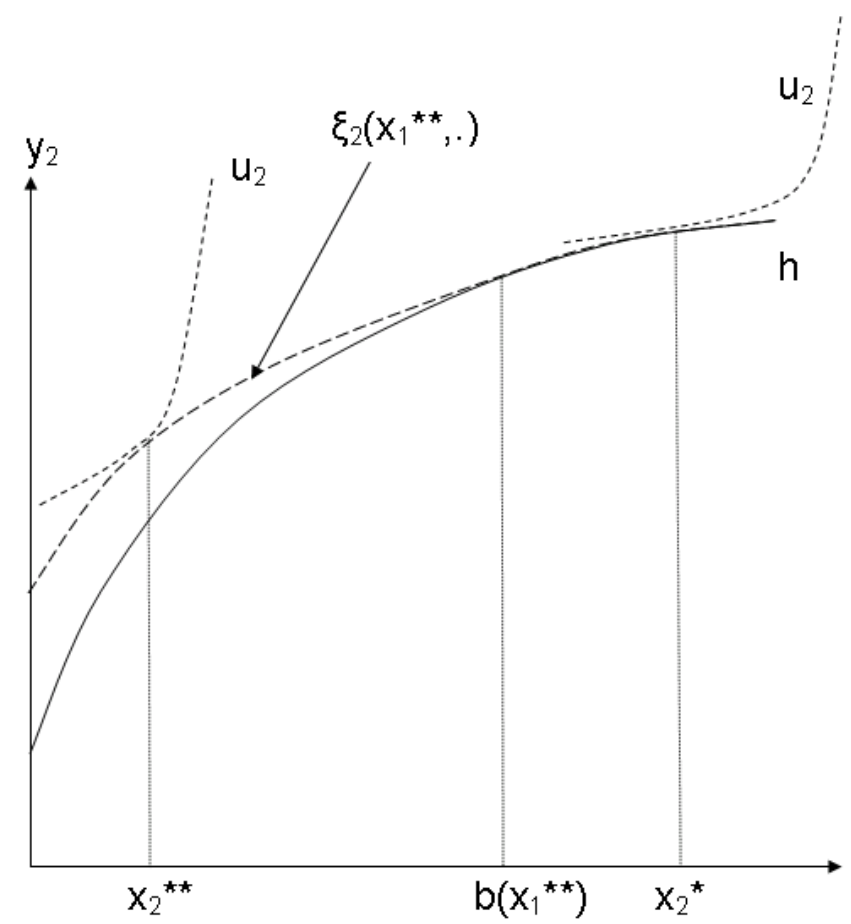

Figure 1: An impossible configuration.

Claim 1 a) $\xi$ can be written as

$$
\xi(x)=\left(g\left(x_{1}\right), F\left(x_{1}+x_{2}\right)-g\left(x_{1}\right)\right)
$$

on any connected open subset of $S W$ for some mapping $g \in \mathcal{F}$.

b) $\xi$ can be written as

$$
\xi(x)=\left(F\left(x_{1}+x_{2}\right)-h\left(x_{2}\right), h\left(x_{2}\right)\right)
$$

on any connected open subset of $N E$ for some mapping $h \in \mathcal{F}$.

Proof. We only prove statement a). By definition, $\frac{\partial \xi_{1}}{\partial x_{2}} \equiv 0$ on $S W$, which implies that for any connected open subset, $\Sigma$, of $S W$ there exists a mapping $g$ such that $\xi_{1}(x)=g\left(x_{1}\right)$ on $\Sigma$; also, budget balance requires $\xi_{2}(x)=F\left(x_{1}+x_{2}\right)-g\left(x_{1}\right)$ on $\Sigma$. The monotonicity and smoothness of $\xi$ imply that $g$ must be strictly increasing and continuously differentiable, respectively.

It remains to show the strict concavity of $g$. Consider any $x \in \Sigma$ and $\varepsilon>0$ such that the closed ball, $\bar{B}(x, \varepsilon)$, is included in $\Sigma$ and suppose $g$ is convex on $\left[x_{1}-\varepsilon, x_{1}+\varepsilon\right]$. Because for any $s \in\left[x_{1}-\varepsilon, x_{1}+\varepsilon\right], \xi_{2}(s, \cdot) \equiv F(s+\cdot)-g(s)$ is strictly concave, one can find a utility function $u_{2}$ such that $x_{2}$ is agent 2's best response to any $s \in\left[x_{1}-\varepsilon, x_{1}+\varepsilon\right] .{ }^{5}$ Then, one can construct a utility function $u_{1}$ such that $u_{1}\left(x_{1}-\varepsilon, g\left(x_{1}-\varepsilon\right)\right)=u_{1}\left(x_{1}+\varepsilon, g\left(x_{1}+\varepsilon\right)\right)=\max _{\left[x_{1}-\varepsilon, x_{1}+\varepsilon\right]}\left\{u_{1}(s, g(s))\right\}$; see Figure 2. By monotonicity of $\xi_{1}\left(\cdot, x_{2}\right)$ and convexity of preferences, one can find $u_{1}$ "convex enough" such that $u_{1}\left(x_{1}-\varepsilon, g\left(x_{1}-\varepsilon\right)\right)=u_{1}\left(x_{1}+\varepsilon, g\left(x_{1}+\varepsilon\right)\right)=\max _{\left\{s \mid\left(s, x_{2}\right) \in \Sigma\right\}}\left\{u_{1}\left(s, \xi_{1}\left(s, x_{2}\right)\right)\right\}$. Hence, both $\left(x_{1}-\varepsilon, x_{2}\right)$ and $\left(x_{1}+\varepsilon, x_{2}\right)$ are Nash equilibria of the supply game, contradicting the

\footnotetext{
${ }^{5}$ E.g. by making the indifference curves of agent 2's preference arbitrarily close to being right-angled.
} 


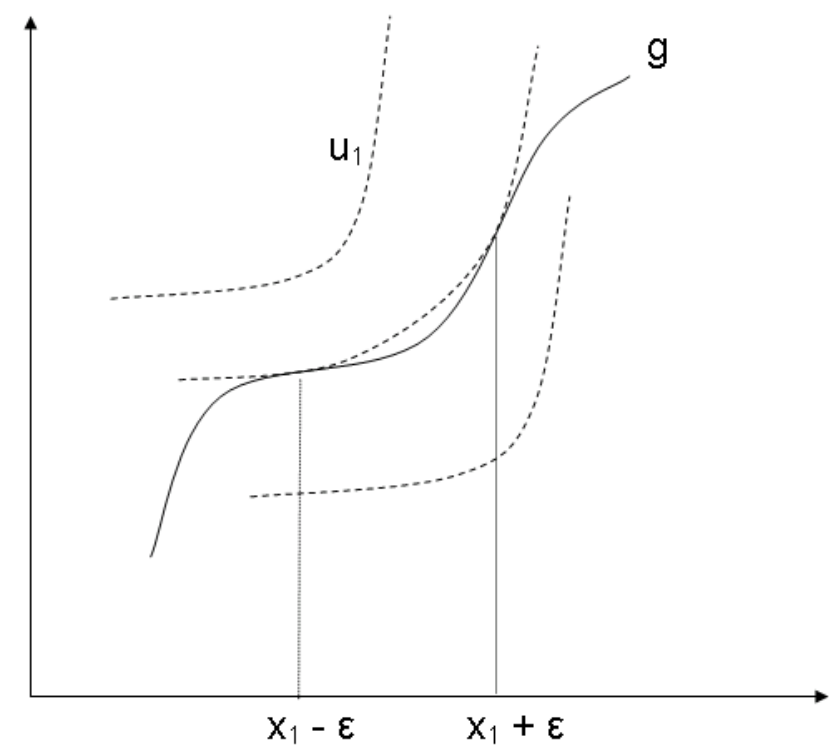

Figure 2: Multiple equilibria may exist if $g$ is not strictly concave.

uniqueness assumption.

The remainder of the proof consists in establishing that the boundary between $N E$ and $S W$ is increasing and is the graph of some increasing real-valued function. But first we must make sure that this boundary exists.

Claim 2 i) $D$ has empty interior,

ii) there exists a boundary, $B$, between $N E$ and $S W$, if both are nonempty,

iii) $B \subset D$.

Proof. Suppose i) is false and consider an open neighborhood in $D$ containing 4 points $x^{A}, x^{B}, x^{C}$, $x^{D}$ such that $x^{C}\left(\operatorname{resp} . x^{D}\right)$ lies North of $x^{A}\left(\right.$ resp. $\left.x^{B}\right)$ and $x^{B}\left(\right.$ resp. $\left.x^{D}\right)$ lies East of $x^{A}$ (resp. $\left.x^{C}\right)$; see Figure 3 .

Notice that budget balance requires $\xi_{1}(x)+\xi_{2}(x)=F\left(x_{1}+x_{2}\right)$ for all $x$, which implies:

$$
\frac{\partial \xi_{1}}{\partial x_{1}}(x)+\frac{\partial \xi_{2}}{\partial x_{1}}(x)=F^{\prime}\left(x_{1}+x_{2}\right)
$$

for all $x \in \mathbb{R}_{+}^{2}$. Therefore, $\frac{\partial \xi_{1}}{\partial x_{1}}(x)=F^{\prime}\left(x_{1}+x_{2}\right)$ on $D$. Thus, taking the integral between $x^{A}$ and $x^{B}$ yields $\xi_{1}\left(x^{B}\right)=\xi_{1}\left(x^{A}\right)+F\left(x_{1}^{B}+x_{2}^{B}\right)-F\left(x_{1}^{A}+x_{2}^{A}\right)$; also, $\xi_{1}\left(x^{D}\right)=\xi_{1}\left(x^{B}\right)$ because $\frac{\partial \xi_{2}}{\partial x_{1}} \equiv 0$ on $D$. Therefore $\xi_{1}\left(x^{D}\right)=\xi_{1}\left(x^{A}\right)+F\left(x_{1}^{B}+x_{2}^{B}\right)-F\left(x_{1}^{A}+x_{2}^{A}\right)$. Similarly, $\xi_{1}\left(x^{D}\right)=$ $\xi_{1}\left(x^{C}\right)+F\left(x_{1}^{D}+x_{2}^{D}\right)-F\left(x_{1}^{C}+x_{2}^{C}\right)=\xi_{1}\left(x^{A}\right)+F\left(x_{1}^{D}+x_{2}^{D}\right)-F\left(x_{1}^{C}+x_{2}^{C}\right)$, which implies

$$
F\left(x_{1}^{B}+x_{2}^{B}\right)-F\left(x_{1}^{A}+x_{2}^{A}\right)=F\left(x_{1}^{D}+x_{2}^{D}\right)-F\left(x_{1}^{C}+x_{2}^{C}\right),
$$

a clear contradiction of the strict concavity of $F$. Therefore $D$ is of empty interior and the boundary between $N E$ and $S W$ exists; smoothness implies that the latter is contained in $D$. 


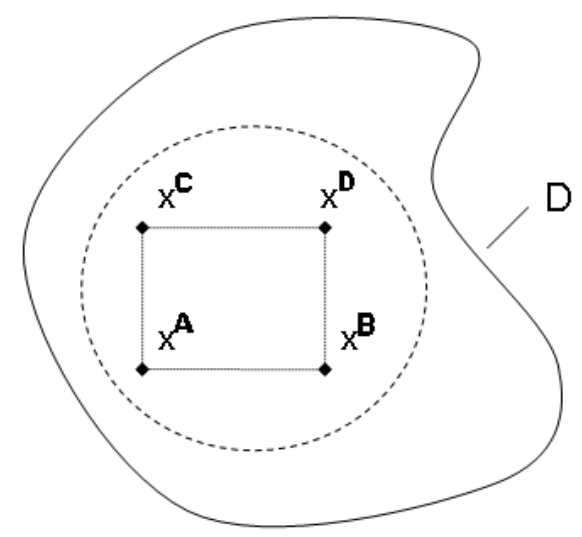

Figure 3:

We call any continuous path of $\mathbb{R}_{+}^{2}$ which is a subset of $B$ a portion of the boundary.

Claim $3 B$ does not contain vertical or horizontal portions.

Proof. Suppose $B$ contains a non-degenerate horizontal portion $\left[x_{1}^{-}, x_{1}^{+}\right] \times\left\{x_{2}\right\}$. Smoothness, along with the fact that $B \subset D$ and Claim 1 imply $h^{\prime}\left(x_{2}\right)=g^{\prime}\left(x_{1}\right)$ for all $x_{1}$ in some non-degenerate subinterval of $\left[x_{1}^{-}, x_{1}^{+}\right]$for some mappings $g, h \in \mathcal{F}$, contradicting the strict concavity of $g$. Similarly, $B$ cannot contain a vertical portion.

Claim 4 On a portion of $B, x_{2}$ increases with $x_{1}$.

Proof. As above, $h^{\prime}\left(x_{2}\right)=g^{\prime}\left(x_{1}\right)$ must hold at any point on the boundary and the claim holds true by strict concavity of $g$ and $h$.

We introduce some more notation. We define the sets $S W^{+}=\left\{x \in S W \mid \frac{\partial \xi_{2}(x)}{\partial x_{1}}>0\right\}$ and $S W^{-}=$ $\left\{x \in S W \mid \frac{\partial \xi_{2}(x)}{\partial x_{1}}<0\right\}$; the sets $N E^{+}$and $N E^{-}$are similarly defined. By smoothness, these four sets are open.

Claim 5 a) $S W^{-}$is north-comprehensive: $S W^{-}+\{0\} \times \mathbb{R}_{+} \subset S W^{-}$,

b) $S W^{+}$is south-comprehensive: $S W^{+}+\{0\} \times \mathbb{R}_{-} \subset S W^{+}$,

c) $N E^{-}$is east-comprehensive: $N E^{-}+\mathbb{R}_{+} \times\{0\} \subset N E^{-}$,

d) $N E^{+}$is west-comprehensive: $N E^{+}+\mathbb{R}_{-} \times\{0\} \subset N E^{+}$.

Proof. We only prove statement a). Let $x \in S W^{-}$, and consider an open neighborhood of $x$ contained in $S W^{-}$. On that neighborhood, $\frac{\partial \xi_{1}}{\partial x_{2}}=0$, i.e. $\xi_{1}$ is independent of $x_{2}$; in particular, the ratio $\frac{\xi_{1}\left(x_{1}+\varepsilon, x_{2}\right)-\xi_{1}\left(x_{1}, x_{2}\right)}{\varepsilon}$ is also independent of $x_{2}$ on that neighborhood for small values of $\varepsilon$. Taking the limit, $\frac{\partial \xi_{1}}{\partial x_{1}}$ is independent of $x_{2}$ on a neighborhood of $x$. By the strict concavity of $F$, expression (1) implies that $\frac{\partial \xi_{2}}{\partial x_{1}}$ must be decreasing in $x_{2}$. In addition, because $x \in S W^{-}$, we have $\frac{\partial \xi_{2}}{\partial x_{1}}(x)<0$; it follows that $\frac{\partial \xi_{2}}{\partial x_{1}}\left(x_{1}, x_{2}+\lambda\right)<0$ for any $\lambda>0$. Thus, by acyclicity, $S W^{-}$is north-comprehensive. 


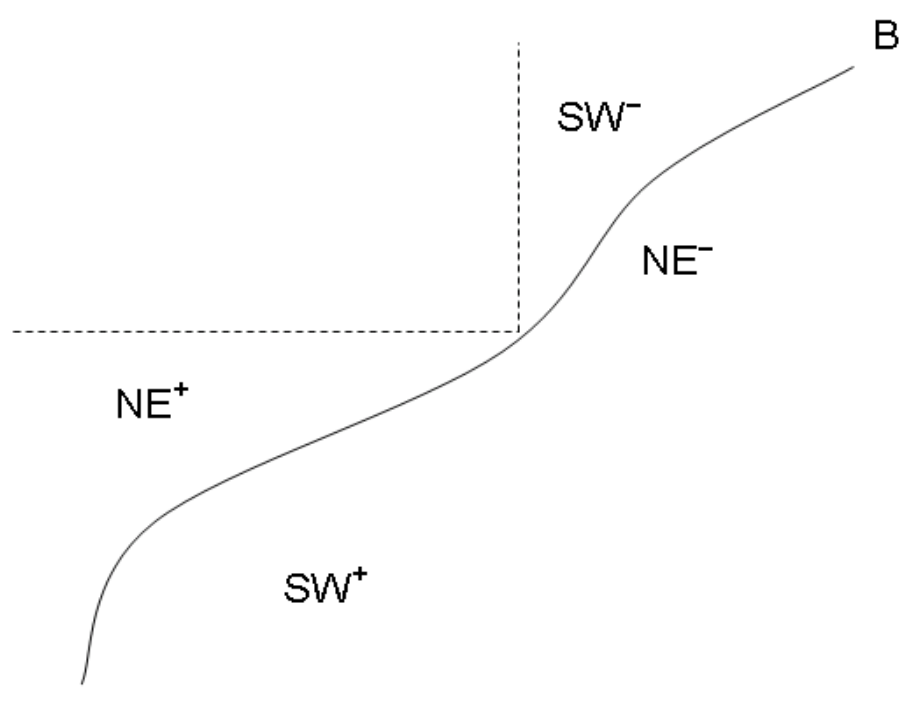

Figure 4:

More notation. We denote by $S W^{-} / N E^{-}$a portion of $B$ with $S W^{-}$(resp. $N E^{-}$) in the immediate northwest (resp. southeast) vicinity of the boundary. $N E^{+} / S W^{+}$portions are similarly defined.

Claim $6 B$ consists only of $S W^{-} / N E^{-}$and $N E^{+} / S W^{+}$portions.

Proof. From the previous claim. Because $S W^{-}$is north-comprehensive and because $B$ is included in $D$, there cannot be any points in $S W^{-}$south of $B$. Similarly, there cannot be any points in $S W^{+}$ (resp. $N E^{-}, N E^{+}$) north (resp. west, east) of $B$.

We complete the proof of the claim by showing that any portion of $B$ containing a $S W^{-} / N E^{-}$ (resp. $N E^{+} / S W^{+}$) subportion must be a $S W^{-} / N E^{-}$(resp. $N E^{+} / S W^{+}$) portion. Suppose there exists a portion of $B$ containing both a $S W^{-} / N E^{-}$and a $N E^{+} / S W^{+}$subportion. By the comprehensiveness of $S W^{-}$and $N E^{+}$, it must be that the $S W^{-} / N E^{-}$subportion lies to the northeast of the $N E^{+} / S W^{+}$subportion (see Figure 4). Yet, the north-comprehensiveness of $S W^{-}$and the west-comprehensiveness of $N E^{+}$imply that there exists a horizontal, vertical or decreasing portion of $B$; a contradiction (Claim 4 ).

Claim 7 No point of $B$ can lie northwest of another.

Proof. Suppose the claim is not true and let $x, x^{\prime} \in B$ such that $x_{1}^{\prime}>x_{1}$ and $x_{2}^{\prime}<x_{2}{ }^{6}$ The reader can check that by Claim 5,x and $x^{\prime}$ belong to two portions of different type. W.l.o.g. assume $x$ belongs to a $N E^{+} / S W^{+}$portion and $x^{\prime}$ belongs to a $S W^{-} / N E^{-}$portion.

Define $\bar{x}_{1}>x_{1}$ to be the smallest real number such that $\left(\bar{x}_{1}, t\right) \notin S W^{+}$for any $t \geq 0$ if such a number exists; if not, define $\bar{x}_{1}=x_{1}^{\prime}$. Denote by $b:\left[x_{1}, \bar{x}_{1}\left[\rightarrow \mathbb{R}_{+}\right.\right.$the function whose graph is the north boundary of $S W^{+}$. Note that by the south-comprehensiveness of $S W^{+}, b$ is well defined on $\left[x_{1}, \bar{x}_{1}[\right.$; and by the smoothness of $\xi, b$ is continuous.

\footnotetext{
${ }^{6}$ Clearly, Claim 5 implies $x_{1}^{\prime} \neq x_{1}$ and $x_{2}^{\prime} \neq x_{2}$.
} 
We show that $b$ defines a $N E^{+} / S W^{+}$portion on every interval where it is increasing. Indeed, suppose there exists $\left.x_{1}^{*} \in\right] x_{1}, \bar{x}_{1}$ [ and $\varepsilon>0$ such that the immediate vicinity north of the graph of $b, \mathcal{V} \equiv B\left(\left(x_{1}^{*}, b\left(x_{1}^{*}\right)\right), \varepsilon\right) \cap\left\{x \mid x_{1} \in\left[x_{1}, \bar{x}_{1}\left[, x_{2}>b\left(x_{1}\right)\right\}\right.\right.$, does not intersect $N E^{+}$; i.e., such that

$$
\mathcal{V} \cap N E^{+}=\emptyset
$$

Then claims 5 and 6 imply $\mathcal{V} \subset S W^{-}$, which leads to a contradiction by implying the existence of a horizontal, vertical or decreasing portion of $B$ as in the proof of the previous claim.

We now show that $b$ must be increasing on its domain. Indeed, the immediate vicinity north of the graph of $b$ cannot intersect $N E^{+}$on a non-degenerate non-increasing interval of $b$ (Claim 4), nor can it intersect $N E^{-}$(by east-comprehensiveness of $N E^{-}$, north-comprehensiveness of $S W^{-}$and the fact that $x^{\prime}$ belongs to a $S W^{-} / N E^{-}$portion) or $S W^{+}$(by definition of $b$ ); hence it is a subset of $S W^{-}$, thus leading to the same contradiction as in the previous paragraph.

Finally, because $b$ is increasing on $\left[x_{1}, \bar{x}_{1}\left[, \bar{x}_{1}\right.\right.$ is indeed the smallest number such that $\left(\bar{x}_{1}, t\right) \notin$ $S W^{+}$for any $t \geq 0$ (the north-comprehensiveness of $S W^{-}$implies this fact even if $\bar{x}_{1}$ was originally taken to be equal to $\left.x_{1}^{\prime}\right)$. It follows that a subset of $\left\{\bar{x}_{1}\right\} \times \mathbb{R}_{+}$belongs to the boundary of $S W^{+}$, contradicting the fact that $S W^{+}$cannot have a vertical boundary (easily proved, as in Claim 3 ).

Claim $8 B$ is the graph of a non-decreasing function $b: \mathbb{R}_{+} \rightarrow \mathbb{R}_{+} \cup\{+\infty\}$ which is continuous and increasing on $\{t \geq 0 \mid 0<b(t)<+\infty\}$.

Proof. If $N E=\emptyset$ or $S W=\emptyset$, the boundary $B$ is vacuously defined: set $b \equiv 0$ or $b \equiv+\infty$.

If $N E \neq \emptyset$ and $S W \neq \emptyset$, define the set $X_{1}=\left\{x_{1} \in \mathbb{R}_{+} \mid \exists x_{2}>0\right.$ s.t. $\left.\left(x_{1}, x_{2}\right) \in B\right\}$. By the previous claims $(2,4$ and 7$), X_{1}$ is an interval and there exists a continuous and increasing function $b: X_{1} \rightarrow \mathbb{R}_{+}$whose graph is $B \cap\left(X_{1} \times \mathbb{R}_{+}\right)$. We extend the domain of $b$ by setting $b\left(x_{1}\right)=0$ for all $x_{1} \leq \inf X_{1}$ if $\inf X_{1}>0$ and by defining $b\left(x_{1}\right)=+\infty$ for all $x_{1} \geq \sup X_{1}$ if $\sup X_{1}$ exists.

Conclusion of the proof: The statement of Theorem 1 follows from claims 1 and 8 .

\section{References}

[1] Alcalde, J., Angel Silva, J., 2004. A proposal for sharing costs. Journal of Mathematical Economics 40, 831-845.

[2] Corchón, L.C., Puy, M.S., 2002. Existence and Nash implementation of efficient sharing rules for a commonly owned technology. Soc. Choice Welfare 19, 369-379.

[3] Dasgupta, P., Hammond, P., Maskin, E., 1979. The implementation of social choice rules: Some results on incentive compatibility. Rev. Econ. Stud. 46, 185-216.

[4] Friedman, E. J., 2002. Strategic properties of heterogeneous serial cost sharing. Mathematical Social Sciences 44, 145-154.

[5] Friedman, E.J., 2004. Strong monotonicity in surplus sharing. Econ. Theory 23, 643-658.

[6] de Frutos, M.A. 1998. Decreasing Serial Cost Sharing under Economies of Scale. J. Econ. Theory 79, 245-275. 
[7] Hougaard, J. L., Thorlund-Petersen, L., 2000. The stand-alone test and decreasing serial cost sharing. Economic Theory 16, 355-362.

[8] Israelsen, D., 1980. Collectives, Communes, and Incentives. J. Compar. Econ. 4, 99-124.

[9] Leroux, J., 2004. Strategyproofness and efficiency are incompatible in production economies. Economics Letters 85, 335-340.

[10] Leroux, J., 2005 Strategy-proof profit sharing in partnerships: Improving upon autarky, Soc. Choice Welfare, forthcoming.

[11] Moulin, H., 2002. "Axiomatic Cost and Surplus-Sharing," in Handbook of Social Choice and Welfare vol.1 (Arrow, Sen, and Suzumura Eds.) pp.289-357, North-Holland.

[12] Moulin, H., Shenker, S., 1992. Serial Cost Sharing. Econometrica 60(5), 1009-1037.

[13] Moulin, H., Watts, A., 1997. Two versions of the tragedy of the common. Economic Design 2, $399-421$.

[14] Satterthwaite, M. A., Sonnenschein, H., 1981. Strategy-Proof Allocations at Differentiable Points. Review of Economic Studies 48, 587-597.

[15] Sen, A.K., 1966. Labour allocation in a cooperative enterprise. Rev. Econ. Stud. 33, 361-371.

[16] Shenker, S., "On the strategy-proof and smooth allocation of private goods in a production economy", mimeo, Xerox PARC, 1992.

[17] Shin, S., Suh, S-C, 1997. Double Implementation by a Simple Game Form in the Commons Problem. Journal of Economic Theory 77, 205-213.

[18] Sprumont, Y., 1998. Ordinal Cost Sharing. Journal of Economic Theory 81, 126-162.

[19] Suh, S-C., "Two Serial Mechanisms in a Surplus Sharing Problem," mimeo, University of Windsor, 1997.

[20] Téjédo, C., Truchon, M., 2002. Serial cost sharing in multidimensional contexts. Mathematical Social Sciences 44, 277-299.

[21] Weitzman, M., 1974. Free access vs private ownership as alternative systems for managing common property. J. Econ. Theory 8, 225-234. 


\section{Liste des cahiers de recherche publiés par les professeurs 2005-2006}

\section{Institut d'économie appliquée}

IEA-05-01 DÉSIRÉ VENCATACHALLUM ET BRUNO VERSAEVEL. « R\&D Delegation in a Duopoly with Spillovers », 57 pages.

IEA-05-02 MiCHEL NORMANDIN ET PASCAL ST-AMOUR.. « An Empirical Analysis of U.S. Aggregate Portfolio Allocations », 33 pages.

IEA-05-03 MARTIN BOILEAu ET MiCHEL nORMANDin. « Closing International Real Business Cycle Models with Restricted Financial Markets », 36 pages.

IEA-05-04 GEORGES DIONNE ET BENOIT DOSTIE. «New Evidence on the Determinants of Absenteeism Using Linked Employer-Employee Data », 35 pages.

IEA-05-05 MAURICE N. MARCHON. «Perspectives économiques canadiennes dans un contexte international », 27 pages.

IEA-05-06 RAYNAULD, JACQUES. «L'efficacité de l'apprentissage en ligne: le cas d'un cours d’économie à HEC Montréal », 10 pages

IEA-05-07 MICHEL NORMANDIN ET BRUNO POWO FOSSO. «Global versus Country-Specific Shocks and International Business Cycles», 44 pages.

IEA-05-08 RAYNAULD, JACQUES. « Le programme Virtuose : les ordinateurs portatifs et l'intégration des TIC à HEC Montréal - 1996-2001 », 88 pages 
IEA-06-01 DOSTIE, BENOIT ET LÉGER PIERRE THOMAS. « Self-selection in migration and returns to unobservable skills », 88 pages

IEA-06-02 JÉRÉMY LAURENT-LUCCHETTI AND ANDREW LEACH. «Induced innovation in a decentralized model of climate change », 34 pages.

IEA-06-03 BENOIT DOSTIE, RAJSHRI JAYARAMAN AND MATHIEU TRÉPANIER. « The Returns to Computer Use Revisited, Again », 27 pages.

IEA-06-04 MICHEL NORMANDIN. « The Effects of Monetary-Policy Shocks on Real Wages: A MultiCountry Investigation », 38 pages.

IEA-06-05 MICHEL NORMANDIN. «Fiscal Policies, External Deficits, and Budget Deficits », 50 pages.

IEA-06-06 J. DAVID CUMMINS, GEORGES DIONNE, ROBERT GAGNÉ AND ADBELHAKIM NOUIRA. «Efficiency of Insurance Firms with Endogenous Risk Management and Financial Intermediation Activities », 41 pages.

IEA-06-07 LUC BAUWENS AND JEROEN V.K. ROMBOUTS. «Bayesian Inference for the Mixed Conditional Heteroskedasticity Model », 25 pages.

IEA-06-08 LUC BAUWENS ARIE PREMINGER AND JEROEN V.K. ROMBOUTS. «Regime Switching Garch Models », 25 pages.

IEA-06-09 JEROEN V.K. ROMBOUTS AND TAOUFIK BOUEZMARNI. « Nonparametric Density Estimation for Positive Time Series », 32 pages.

IEA-06-10 JUSTIN LEROUX. «Cooperative production under diminishing marginal returns: Interpreting fixed-path methods », 25 pages. 